\title{
Estudo Estatístico Multivariado da Qualidade do ar em Localidades com Perfil Residencial e Industrial
}

\author{
Taisa C. Dantas, Thainá C. Souza \& Sergio M. Corrêa
}

Este estudo compara a qualidade do ar em dois locais na Região Metropolitana do Rio de Janeiro, um urbano (Taquara- TQ) e um outro industrial (Jardim Primavera JP). Os dados utilizados correspondem as médias horárias de 2014 a 2016 para óxidos nitrogenados ( $\mathrm{NOx}, \mathrm{NO}_{2}$ e $\left.\mathrm{NO}\right)$, ozônio $\left(\mathrm{O}_{3}\right)$, monóxido de carbono $(\mathrm{CO})$, dióxido de enxofre $\left(\mathrm{SO}_{2}\right)$. E material particulado (MP10), assim como dados meteorológicos. Para analisar os resultados, uma abordagem estatística multivariada foi aplicada usando a Linguagem R. Os resultados indicam que existe uma possível influência da REDUC na região do Jardim Primavera, devido às altas concentrações de $\mathrm{MP} 10, \mathrm{SO}_{2}$ e $\mathrm{O}_{3}$ comparado com a Taquara, a qual tem um grande efeito das fontes móveis que geram NOx e CO, evidenciado pela alta concentração desses compostos.

Palavras Chave: Linguagem R; Qualidade do Ar; Poluentes atmosféricos; região Urbana; Região Industrial.

This study compares the air quality of two locations in the Metropolitan Area of Rio de Janeiro, an urban (Taquara - TQ) and an industrial location (Jardim Primavera - JP). The data used correspond to the hourly averages from 2014 to 2016 for nitrogen oxides ( $\mathrm{NOx}, \mathrm{NO}_{2}$ and $\mathrm{NO}$ ), ozone $\left(\mathrm{O}_{3}\right)$, carbon monoxide $(\mathrm{CO})$, sulfur dioxide $\left(\mathrm{SO}_{2}\right)$, and particulate matter (PM10) as well as meteorological data. To analyze the results, a multivariate statistic approach was applied using R Language. The results indicate that there is a possible influence of REDUC in the Jardim Primavera location due to the high concentrations of PM10, $\mathrm{SO}_{2}$ and $\mathrm{O}_{3}$ compared to Taquara, which has a great effect of the mobile sources that generates NOx and $\mathrm{CO}$, evidenced by the high concentration of these compounds.

Keywords: R Language; Air Quality; Air Oollutants; Urban Region; Industrial Region. 


\section{Introdução}

A qualidade do ar é o resultado de diversos fatores, tais como as emissões antropogênicas (fontes fixas e fontes móveis) e naturais, da meteorologia e da topografia. $\mathrm{O}$ ar que a população respira depende não só das emissões dos poluentes primários, mas também da conversão destes poluentes em poluentes secundários. Diante disso, com o objetivo de proteger o meio ambiente e a saúde populacional foi promulgada em 1990 a Resolução número 03 do CONAMA que estabelece os padrões primários e secundários de qualidade do ar, dentre quais estão inclusos o ozônio $\left(\mathrm{O}_{3}\right)$, os óxidos de nitrogênio (NOx), monóxido de carbono (CO), dióxido de enxofre ( $\left.\mathrm{SO}_{2}\right)$, e partículas inaláveis (MP10).

Diante disso, as agências ambientais (estaduais e municipais) possuem um campo de atuação que envolve o controle das fontes emissoras (veículos e industrias) e o monitoramento da qualidade do ar. Entretanto, cada localidade apresenta características distintas, que envolvem o perfil socioeconômico, tamanho da população local, presença das diferentes fontes emissoras, da meteorologia e da topografia.

Neste trabalho buscou-se comparar a qualidade do ar durante os anos de 2014 a 2016 de duas estações da qualidade do ar com perfis distintos, uma localizada em uma região predominantemente residencial e com fontes móveis (Taquara) e em outra com perfil industrial e com diversas fontes estacionárias oriundas da Refinaria REDUC (Jardim Primavera).

Com os dados obtidos pelas estações automáticas da qualidade do ar do INEA, foi utilizada a Linguagem $\mathrm{R}$ com o pacote Openair para o estudo estatístico descritivo e multivariado dos dados. Os dados serão apresentados para cada poluente de forma individual em médias horarias ao longo do triênio proposto, para fins de comparação entre as localidades (boxplot e time plot) e com a legislação da Resolução 03/90. Em seguida os dados serão correlacionados entre eles por meio de matrizes de correlação e dendogramas. Também será utilizado um método de hierarquização (Boruta) para avaliar a contribuição de cada parâmetro na formação do O3 troposférico. Os dados dos poluentes também serão comparados e correlacionados com os parâmetros meteorológicos como a temperatura, a umidade relativa, a direção e intensidade do vento, por meio de Annular Plot.

\section{Resultados e Discussão}

A Tabela 1 apresenta um resumo dos dados dos principais poluentes avaliados em uma estação de monitoramento do ar situada em uma área industrial, Jardim Primavera (JP), e em uma área urbana, Taquara (TQ). A tabela também conta com duas colunas que mostram limites de qualidade do ar brasileiros fornecidas pelo MMA (Ministério do Meio Ambiente), Resolução CONAMA 003/1990, e os limites equivalentes norte americanos fornecido pela U.S.EPA (Agência de Proteção Ambiental dos Estados Unidos), o NAAQS. Todos os limites propostos, com exceção dos anuais, não devem ser excedidos mais de uma vez por ano.

Comparando a emissão de $\mathrm{CO}$ com os limites, percebese que ambas as estações possuem medianas dentro dos padrões, já quanto à máxima, a Estação JP ultrapassou o limite ao menos uma vez no triênio, uma vez que esta possui uma máxima de $9,66 \mathrm{ppm}$. O CO tem sua maior parte de emissão em áreas urbanas decorrentes de veículos automotores e o resultado superior da mediana para a TQ confirma o esperado.

Com relação ao $\mathrm{NO}_{2}$ todas as emissões estão dentro do limite, tanto as medianas quanto as máximas de ambas as estações, entretanto é interessante chamar a atenção para o fato da mediana da estação TQ ser maior do que a JP, podendo indicar que a contribuição das emissões veiculares para esse poluente causa um impacto muito maior do que a emissão industrial. O mesmo vale para o NOx, que segue a mesma tendência de possuir valores menores na estação JP do que na TQ.

Para o $\mathrm{SO}_{2}$ é possível reparar que as medianas estão bem abaixo do limite permitido, entretanto a estação JP possui uma máxima que mostra que esta estação excedeu pelo menos uma vez o parâmetro ao longo desses anos. O material particulado também possui uma mediana dentro dos padrões de detecção, contudo as máximas de ambas as estações indicam que este excedeu mais que o dobro do limite nas duas estações por pelo menos uma vez no período analisado. 
Tabela 1. Estatística básica dos dados coletados entre 2014 e 2016 nas estações de monitoramento Jardim Primavera (JP) e Taquara (TQ) e dos limites de emissões.

\begin{tabular}{|c|c|c|c|c|c|c|}
\hline & \multicolumn{2}{|c|}{ Mediana } & \multicolumn{2}{|c|}{ Máximo } & \multirow{2}{*}{ Limite CONAMA } & \multirow{2}{*}{ Limite NAAQS } \\
\hline & JP & TQ & JP & TQ & & \\
\hline $\mathrm{CO}(\mathrm{ppm})$ & 0.431 & 0.630 & 9.657 & 2.680 & $\begin{array}{c}9 \mathrm{ppm}(8 \mathrm{~h}) \\
35 \mathrm{ppm}(1 \mathrm{~h})\end{array}$ & $\begin{array}{l}\text { 9ppm (8h) } \\
35 \text { ppm (1h) }\end{array}$ \\
\hline NO (ppb) & 17.000 & 34.300 & 302.000 & 280.200 & - & - \\
\hline $\mathrm{NO}_{2}(\mathrm{ppb})$ & 14.000 & 28.100 & 158.000 & 139.100 & $\begin{array}{c}170 \mathrm{ppb}(1 \mathrm{~h}) \\
53 \mathrm{ppb}(1 \text { ano })\end{array}$ & $\begin{array}{c}100 \mathrm{ppb}(1 \mathrm{~h}) \\
53 \mathrm{ppb} \text { (1 ano) }\end{array}$ \\
\hline NOx (ppb) & 33.000 & 65.500 & 308.000 & 347.300 & - & - \\
\hline $\mathrm{O}_{3}(\mathrm{ppb})$ & 10.292 & 5.300 & 197.366 & 146.300 & $82 \mathrm{ppb}(1 \mathrm{~h})$ & $70 \mathrm{ppb}(8 \mathrm{~h})$ \\
\hline $\mathrm{SO}_{2}(\mathrm{ppb})$ & 2.144 & 0.400 & 152.757 & 30.399 & $\begin{array}{c}140 \mathrm{ppb}(24 \mathrm{~h}) \\
30,5 \mathrm{ppb}(1 \mathrm{ano})\end{array}$ & $75 \mathrm{ppb}(1 \mathrm{~h})$ \\
\hline $\operatorname{MP} 10(\mu \mathrm{g} / \mathrm{m} 3)$ & 47.000 & 35.000 & 360.000 & 338.000 & $\begin{array}{l}150 \mu \mathrm{g} / \mathrm{m} 3(24 \mathrm{~h}) \\
50 \mu \mathrm{g} / \mathrm{m} 3 \text { (1 ano })\end{array}$ & $150 \mu \mathrm{g} / \mathrm{m} 3(1 \mathrm{~h})$ \\
\hline
\end{tabular}

$\mathrm{O} \mathrm{O}_{3}$ possui medianas dentro dos padrões nas duas estações, porém suas máximas indicam que as emissões excederam o limite pelo menos uma vez nesses três anos. A formação do $\mathrm{O}_{3}$ troposférico ocorre através de reações químicas complexas que acontecem entre os NOx e compostos orgânicos voláteis, na presença de radiação solar. Com os dados obtidos podemos observar que as medianas menores para o os compostos nitrogenados e maiores para o $\mathrm{O}_{3}$ no JP caracterizam que essa reação acontece com mais vigor nesta localidade, enquanto para a TQ boa parte do $\mathrm{NO}_{2}$ sofrerá apenas uma série de reações naturais na presença radiação solar. Dados de ambos os locais também são apresentados a seguir em forma de timeplot (Figuras 1 e 2) e boxplot (Figuras 3 a 12).

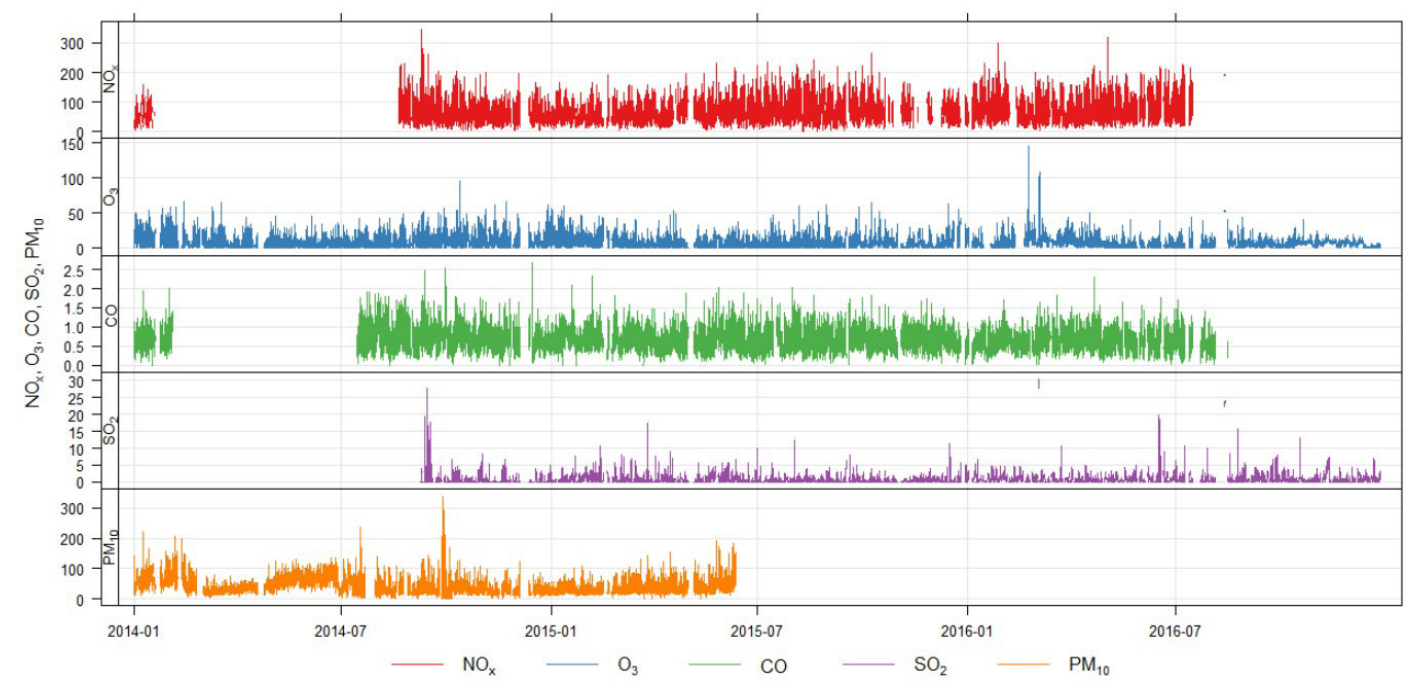

Figura 1. Valores horários médios nos anos de 2014 a 2016 para NOx, $\mathrm{O}_{3}, \mathrm{CO}, \mathrm{SO}_{2}$ e MP10 na TQ. 


\section{Artigo Geral 5}

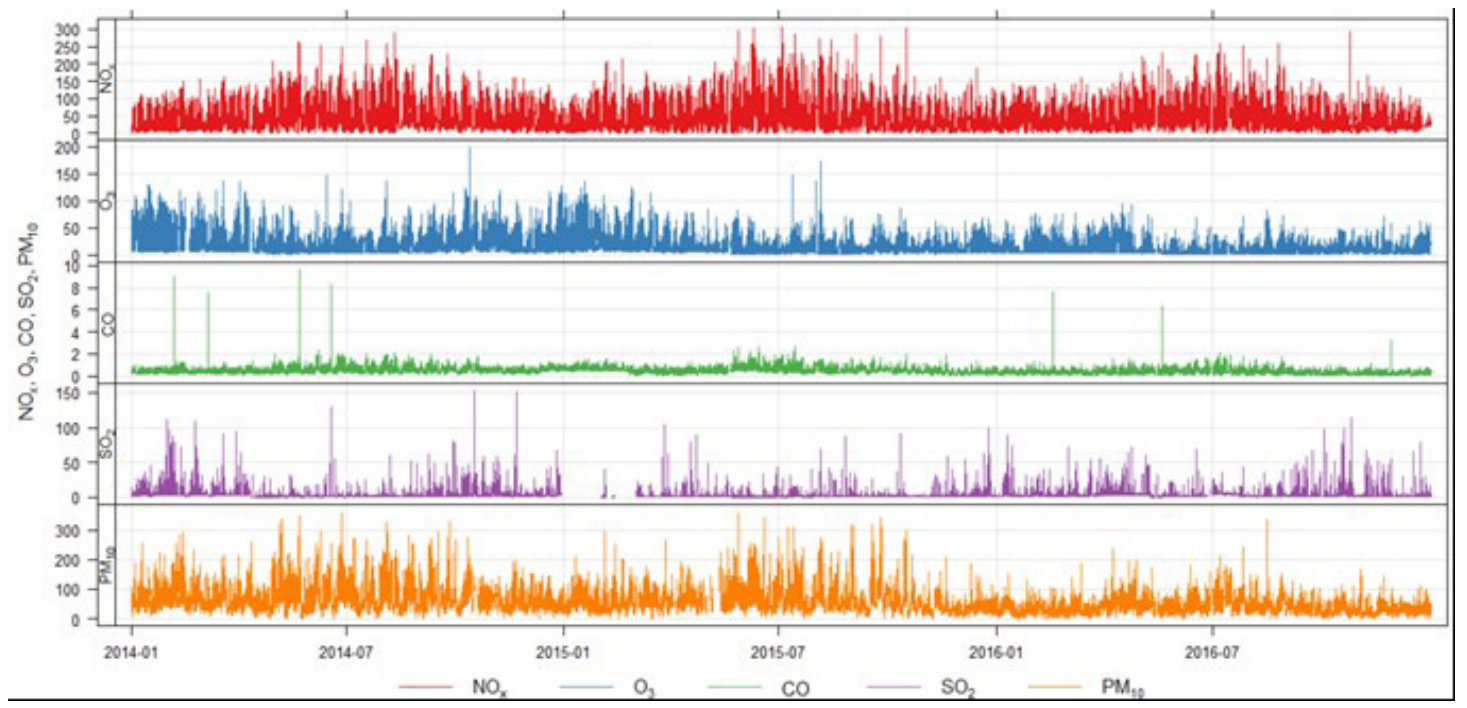

Figura 2. Valores horários médios nos anos de 2014 a 2016 para NOx, $\mathrm{O}_{3}, \mathrm{CO}, \mathrm{SO}_{2}$ e MP10 na JP.

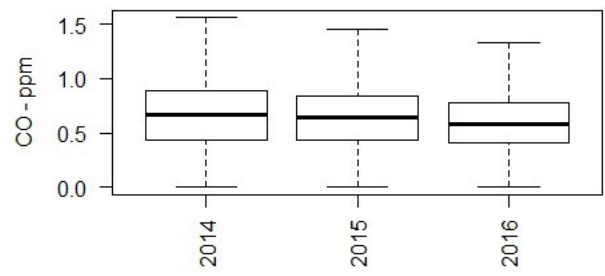

Figura 3. Boxplot para CO TQ



Figura 4. Boxplot para NOx TQ.

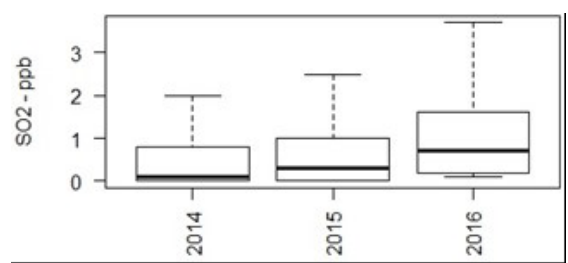

Figura 5. Boxplot para $\mathrm{SO}_{2}$ na TQ.

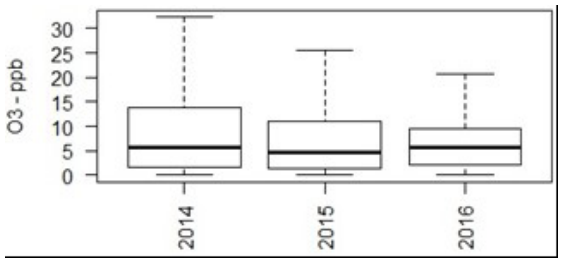

Figura 6. Boxplot para $\mathrm{O}_{3}$ na TQ.

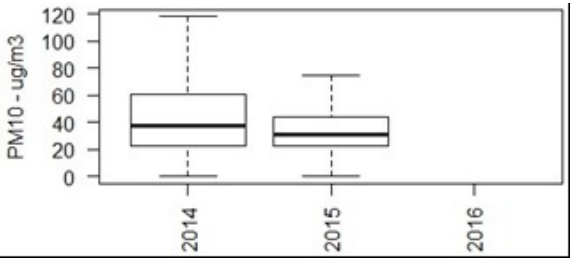

Figura 7. Boxplot para MP10 na TQ.

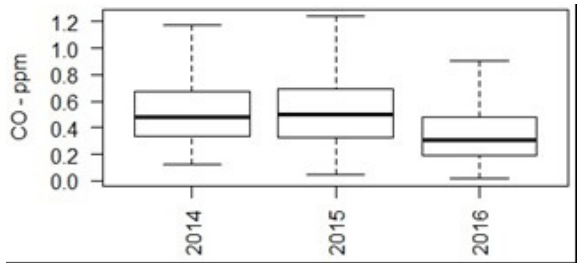

Figura 8. Boxplot para CO na JP. 


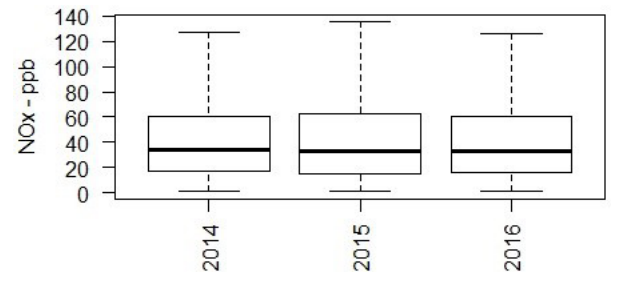

Figura 9. Boxplot para NOx na JP.

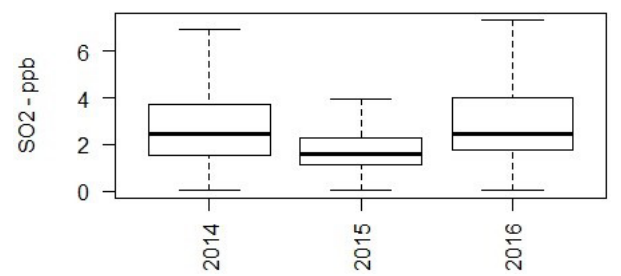

Figura 10. Boxplot para $\mathrm{SO}_{2}$ na JP.



Figura 11. Boxplot para $\mathrm{O}_{3}$ na JP.

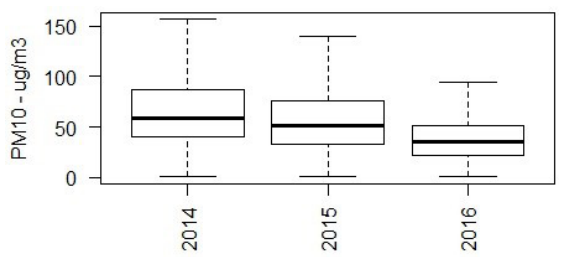

Figura 12. Boxplot para MP10 na JP.

Analisando as figuras 1 e 2 percebe-se com maior clareza quantas vezes o valor limite foi excedido para cada poluente, enquanto os boxplots (Figuras 3 a 12) nos permite verificar a distribuição dos dados. O centro da distribuição é indicado pela linha da mediana, no centro do quadrado. A dispersão é representada pela amplitude do gráfico. O retângulo contém $50 \%$ dos valores do conjunto de dados. A posição da linha mediana no retângulo informa sobre a assimetria da distribuição. Uma distribuição simétrica teria a mediana no centro do retângulo.

Também é fundamental avaliar a influência que cada componente exerce sobre o outro. Dessa forma, faz-se necessária a analise de uma matriz de correlação juntamente com o dendograma.

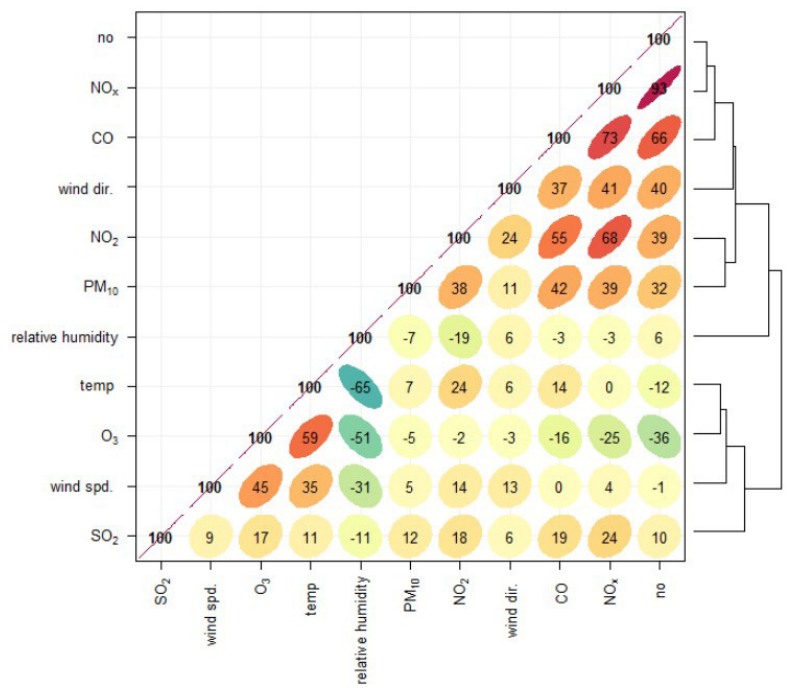

Figura 13. Matriz de correlação com dendograma para TQ.

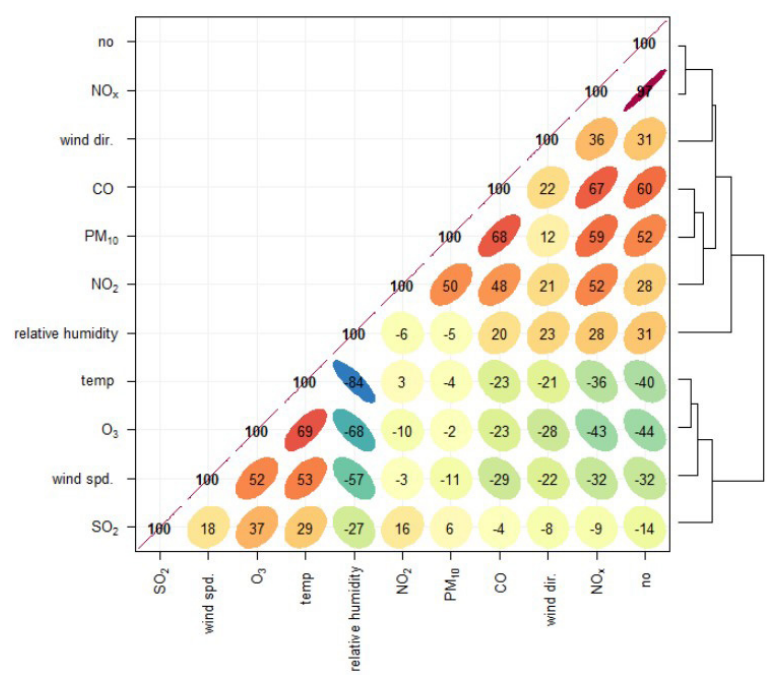

Figura 14. Matriz de correlação com dendograma para JP. 


\section{Artigo Geral 5}

A partir da matriz de correlação pode-se comprovar relações já propostas anteriormente, como a influência do NO e do $\mathrm{NO}_{2}$ para a quantidade de NOx emitido, a forte relação entre o $\mathrm{CO}$ e o NOx uma vez que ambos os compostos são oriundos de fontes móveis de emissão. Também nota-se o elevado efeito da temperatura na formação de $\mathrm{O}_{3}$, uma vez que a produção deste depende da radiação solar, e quanto maior a temperatura, maior a radiação. A relação é inversa entre $\mathrm{O}_{3}$ e umidade relativa, uma vez que o mesmo se forma em dias mais quentes, que tendem a ser mais secos, e consequentemente possuem menor umidade relativa. A relação, também é inversa, entre temperatura e umidade relativa. A relação negativa entre $\mathrm{O}_{3}$ e NO, ocasionada devido ao fato de um composto ser responsável pela formação do outro, sendo assim quanto maior o NO, menor a quantidade de $\mathrm{O}_{3}$ na atmosfera. Percebe-se que o MP10 está relacionado positivamente com os NOx e com o CO.

Os dendogramas associados corroboram as relações estabelecidas pela matriz de correlação. As ligações mais próximas mostram as relações mais positivas entre os compostos e quanto mais afastados indicam relações menos fortes ou inversas entre os poluentes. Por ele pode-se perceber que as maiores relações estão entre o $\mathrm{NO}$ e o NOx, entre o $\mathrm{O}_{3}$ e a temperatura e o MP com o $\mathrm{O}_{3}$.

Outra maneira pela qual se pode confirmar a relação entre $\mathrm{CO}, \mathrm{NO}_{2}$ e $\mathrm{O}_{3}$ é através da analise do Annular Plot que fornece os dados de acordo com os dias da semana.

Como a frota de veículos automotores é reduzida durante os finais de semana, espera-se que haja menor emissão de $\mathrm{NO}_{2}$ e $\mathrm{CO}$, enquanto ocorrerá acumulo de $\mathrm{O} 3$ troposférico. Essa afirmativa é confirmada pelos dados das Figuras 15 e 16.



$\mathrm{ppb}$

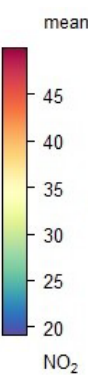

$\mathrm{NO}_{2}$

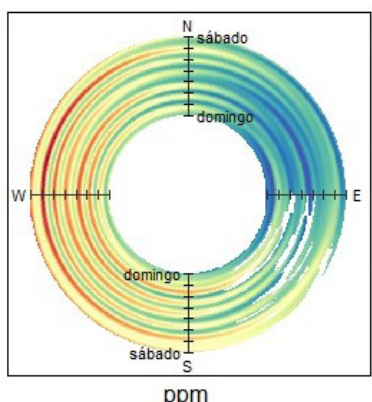

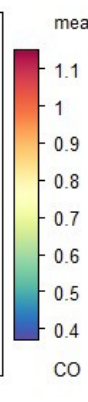

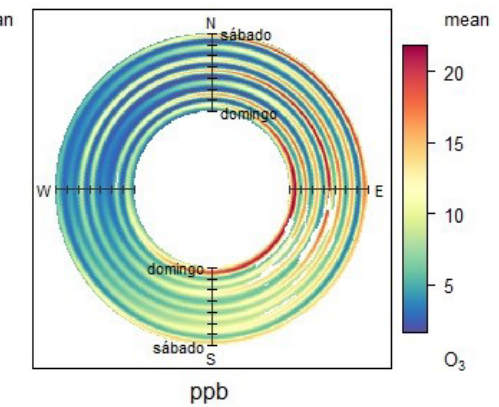

Figura 15: Annular Plot para TQ em função dos dias de semana.

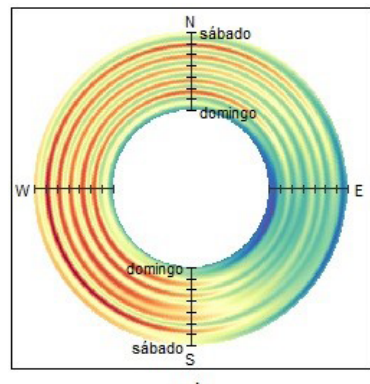

$\mathrm{ppb}$

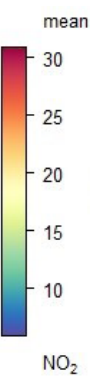

$\mathrm{NO}_{2}$

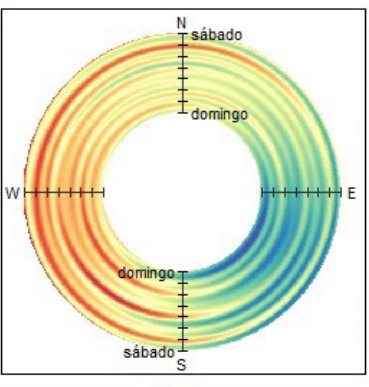

$\mathrm{ppm}$

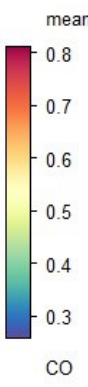

$\mathrm{CO}$

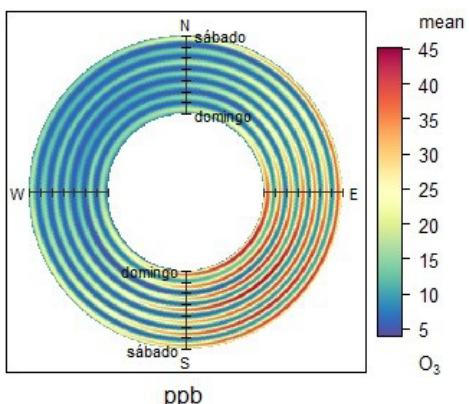

Figura 16. Annular Plot para JP em função dos dias da semana. 
Por fim, utilizou-se o método de classificação Boruta para identificar as principais variáveis relacionadas com a formação de $\mathrm{O}_{3}$ na região urbana e na região industrial. Das Figuras 17 e 18 pode-se perceber que a ordem de importância dos compostos é diferente nas duas regiões analisadas. $\mathrm{Na}$ estação TQ observa-se que as variáveis mais importantes para a formação do $\mathrm{O}_{3}$ são a velocidade e direção do vento, a temperatura e o NO, e a menor importância nessa região é a o $\mathrm{SO}_{2}$. Já na estação JP a variável de maior importância é a data, porém esta é um componente incontrolável. $\mathrm{Na}$ sequência o MP10, a temperatura e o $\mathrm{SO}_{2}$. O maior grau de importância do MP10 e do $\mathrm{SO}_{2}$ para o $\mathrm{O}_{3}$ nessa região indica a presença de uma fonte estacionária próxima à estação mostrando uma possível influência da REDUC na região.

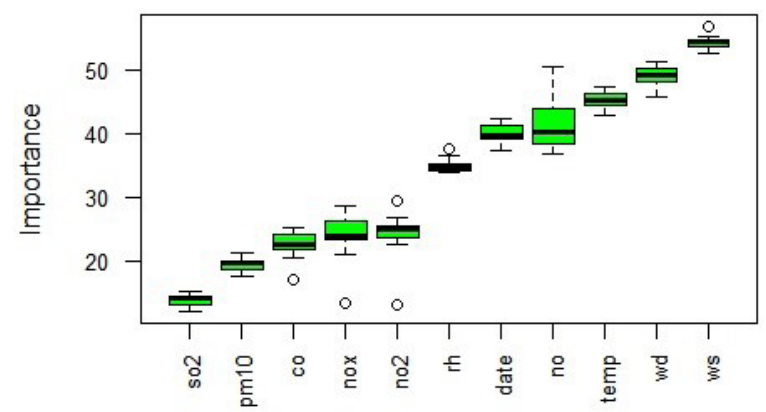

Figura 17. Variáveis de importância para formação de $\mathrm{O}_{3}$ na TQ.

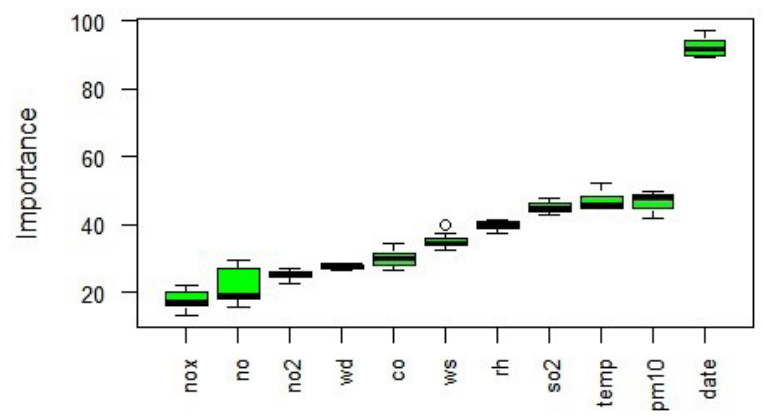

Figura 18. Variáveis de importância para formação de $\mathrm{O}_{3}$ na JP.

\section{Conclusões}

A qualidade do ar em uma região predominantemente residencial e com fontes móveis (Taquara) e em outra com perfil industrial e com diversas fontes estacionárias oriundas da Refinaria REDUC (Jardim Primavera) foi observada e comparada. Alguns poluentes apresentam legislação que os regula, como, por exemplo, $\mathrm{CO}, \mathrm{NO}_{2}, \mathrm{O}_{3}, \mathrm{SO}_{2}$ e MP10.

Para os compostos analisados, a mediana para a TQ apresentou valores superiores ao JP apenas para os NOx e CO. A analise do gráfico Boruta, juntamente com o conceito estabelecido de que o $\mathrm{O}_{3}$ é formado por reações complexas entre NOx e compostos orgânicos voláteis, corroboram o elevado índice de $\mathrm{O} 3$ e a hipótese de que haja um fonte fixa de $\mathrm{SO}_{2}$ nas proximidades da estação JP.

As correlações apresentadas por ambas as localidades foram muito parecidas, sendo as mais fortes entre $\mathrm{NO}$ e o NOx, $\mathrm{O}_{3}$ e a temperatura e MP10 com o $\mathrm{O}_{3}$.

O Annular Plot possibilitou comprovar que os valores altos de ozônio nos fins de semana foram encontrados, provavelmente devido à redução da frota circulante de automóveis, que são grandes emissores de $\mathrm{NO}$, e por esta molécula consomir $\mathrm{O}_{3}$.

\section{Agradecimentos}

Ao CNPq pelo suporte financeiro ao projeto e a bolsa de IT e a FAPERJ pela bolsa de IC.

\section{Referências Bibliográficas}

1. CONAMA. Resolution $\mathrm{N}^{\circ} 03$ of 28 June, 1990. Sets standards of primary and secondary air quality and even the criteria for acute episodes of air pollution. Official Journal of the Federative Republic of Brazil, Brasilia, DF, 23 out. 1990.

2. Gioda, A., Oliveira, R.C.G., Cunha, C.L., Corrêa, S.M., 2017. Understanding ozone formation at two islands of Rio de Janeiro, Brazil. Atmospheric Pollution Research in press. doi.org/10.1016/j. apr.2017.10.003 INEA. http://200.20.53.7/qualiar/home/index. Acesso em Novembro 2017-11-10

3. MMA. Ministério do Meio Ambiente. URL http://www.mma.gov. br/cidades-sustentaveis/qualidade-do-ar/poluentes-atmosféricos. Acesso: 2017.11.09 


\section{Artigo Geral 5}

4. NAAQS. National Ambient Air Quality Standards (40 CFR part 50). URL https://www.epa.gov/criteria-air-pollutants/naaqs-table. Acesso: 2017.11.11

5. R Core Team (2016) R: A language and environment for statistical computing. R Foundation for Statistical Computing, Vienna, Austria. URL https://www.R-project.org/.

\section{Taisa C. Dantas*, Thainá C. Souza \& Sergio M. Corrêa}

Universidade do Estado do Rio de Janeiro, Rodovia Presidente Dutra, km 298, Resende, RJ, Brasil, 27537-000

*E-mail: taisadantasma@gmail.com 